\title{
Dynamic Electrophoretic Mobility of a Concentrated Dispersion of Particles with a Charge-Regulated Surface at Arbitrary Potential
}

\author{
Eric Lee, Chi-Hua Fu, and Jyh-Ping Hsu ${ }^{1}$ \\ Department of Chemical Engineering, National Taiwan University, Taipei, Taiwan 10617, Republic of China
}

Received December 3, 2001; accepted February 14, 2002; published online May 15, 2002

\begin{abstract}
The dynamic electrophoretic mobility of a concentrated dispersion of biocolloids such as cells and microorganisms is modeled theoretically. Here, a biological particle is simulated by a particle, the surface of which contains dissociable functional groups. The results derived provide basic theory for the quantification of the surface properties of a biocolloid through an electroacoustic device, which has the merit of making direct measurement on a concentrated dispersion without dilution. Two key parameters are defined to characterize the phenomenon under consideration: the first, $A$, is associated with the $\mathrm{pH}$ of the dispersion, and the second, $B$, is associated with the equilibrium constant of the dissociation reaction of the functional group. We show that if $A$ is large and/or $B$ is small, the surface potential is high, and the effect of doublelayer polarization becomes significant. In this case the dynamic electrophoretic mobility may have a local maximum and a phase lead as the frequency of the applied electric field varies. Due to the hydrodynamic interaction between neighboring particles, the dynamic electrophoretic mobility decreases with the concentration of dispersion. (c) 2002 Elsevier Science (USA)
\end{abstract}

Key Words: dynamic electrophretic mobility; concentrated dispersion; charge-regulated surface; arbitrary potential.

\section{INTRODUCTION}

Electrokinetic theory is one of the basic tools for the description of the behavior of charged entities. It also provides the foundation for the design of the instruments used to quantify their physicochemical properties. The result of Smoluchoski for electrophoresis (1), for example, is often used to estimate the charged conditions of the surface of an entity. This result was also extended by many researchers to more general cases, which take the effects of practical significance into account (2-5). These include, for example, double-layer polarization (2), arbitrary double-layer thickness and arbitrary surface potential $(4,5)$, and double-layer overlapping and dynamic applied electric field (6-26).

An electroacoustic instrument is a powerful device developed for direct measurement of the properties of a concentrated

\footnotetext{
${ }^{1}$ To whom correspondence should be addressed. Fax: 886-2-23623040. E-mail: jphsu@ccms.ntu.edu.tw.
}

dispersion (6). In contrast to the static electric field applied in the classic electrophoresis measurement, an alternating electric field is applied, and the electrophoretic behavior observed is of a dynamic nature. The result based on electroacoustic measurement is found to be more accurate than that based on the classic method, which involves inevitably some optical limitation (7). The description for the dynamic phenomenon involves solving simultaneously a set of coupled, nonlinear differential equations, which describe electric, flow, and concentration fields. O'Brien (6) was able to derive the dynamic electrophoretic mobility of a dilute dispersion for the case of thin double-layer and low surface potential. His result was extended to more general cases such as arbitrary double-layer thickness and surface potential (8-14). Ohshima (14), for example, investigated the dynamic electrophoretic behavior of a dilute dispersion under the conditions of low surface potential and arbitrary double-layer thickness.

Most of the available theoretical results for dynamic electrophoresis based on an electroacoustic measurement are valid for the case when the volume fraction of the dispersed phase is below $5 \%$. This, however, is unsatisfactory from a practical point of view since dispersion needs to be diluted before its properties can be measured. Apart from a tedious procedure, a diluted dispersion is easier to be affected by various factors such as the concentrations of ionic species and solvent. Therefore, direct measurement on concentrated dispersions is highly desirable, and the corresponding theoretical analysis becomes imminent. Ohshima (15-17) adopted the cell model of Kuwabara (18), in which a dispersion is simulated by a representative particle enclosed by a concentric spherical liquid shell, to analyze the electrophoretic behavior of a concentrated dispersion. The electric potential on a particle surface was assumed to be low, and the boundary condition for electric potential on a cell surface was the same as that adopted by Levine and Neale (19); that is, it is Neumann type. Although the effect of double-layer overlapping was considered, the result obtained still deviated significantly from experimental observations. Dukhin et al. (20) pointed out that the boundary condition used by Shilov and Zharkikh (21), which is Dirichlet type, is more appropriate than that of Levine and Neale (19) for a concentrated dispersion. Lee et al. (22) considered the dynamic electrophoresis of a concentrated dispersion by adopting the boundary condition of Shilov and Zharkikh (21). The effects of double-layer polarization and the overlapping between 
neighboring double layers have been taken into account, and the result obtained is applicable to arbitrary double-layer thickness, surface potential, and the frequency of the applied electric field.

Previous analyses assumed that the surface of a particle remains either at constant potential or at constant surface charge density. These assumptions, although they make the mathematical treatment simpler, can be appropriate for biocolloids such as cells and microorganisms. In a study of the adhesion of biological cells Ninham and Parsegian (27) pointed out that to minimize the total interaction potential, cells are capable of regulating the charged conditions on their surface toward maintaining the interaction surfaces at an ionic equilibrium with the bulk suspension medium. In this case, neither the potential nor the charge density can remain constant during the period of adhesion. Another example is particles covered by an artificial membrane layer where the charged condition depends upon the properties of the latter. The surface of biocolloids usually carry dissociable functional groups. The dissociation of these functional groups yields a charged entity, and the charged condition is a function of the degree of dissociation of the functional groups, which in turn, is affected by factors such as the $\mathrm{pH}$ and the ionic strength of the surrounding medium.

In this study the dynamic electrophoretic mobility of a concentrated dispersion of spherical particles, the surface of which contains dissociable functional groups, is analyzed, taking the effect of double-layer polarization into account. The result derived is capable of describing the electrophoretic behavior of biological particles such as cells and microorganisms. The key factors, which characterize the phenomenon under consideration, including $\mathrm{pH}$ and the equilibrium constant of the dissociation reaction of the functional group, are discussed. The governing equations are solved numerically by a pseudospectral method based on the Chebyshev polynomial, which is justified to be efficient for the present type of problem (22-26).

\section{THEORY}

We consider concentrated, monodispersed, spherical particles in a $z_{1}: z_{2}$ electrolyte solution, $z_{1}$ and $z_{2}$ being respectively the valences of cations and anions with $z_{1}=-\alpha z_{2}$. If $n_{10}$ and $n_{20}$ are the bulk concentrations of cations and anions, then $n_{20}=\left(n_{10} / \alpha\right)$. The surface of a particle contains dissociable functional groups HA, the dissociation of which yields a negatively charged particle. Referring to Fig. 1, the cell model of Kuwabara (18) is adopted to simulate the system under consideration where a representative particle of radius $a$ is enclosed by a concentric liquid shell of radius $b$. The spherical coordinates $(r, \theta, \varphi)$ are adopted with the origin located at the center of the representative particle. We define $H=\lambda^{3}$, with $\lambda=(a / b)$. Then $H$ is an estimate for the volume fraction of particles. An electric field is applied which yields an average electric field $\mathbf{E}=E_{Z} e^{-i \omega t} \mathbf{e}_{Z}$ in the $\pm Z$-direction, where $E_{Z}$ is the magnitude of $\mathbf{E}, i=\sqrt{-1}, \mathbf{e}_{Z}$ is the unit vector in the $Z$-direction, and $\omega$ and $t$ are respectively the frequency of the applied elec-

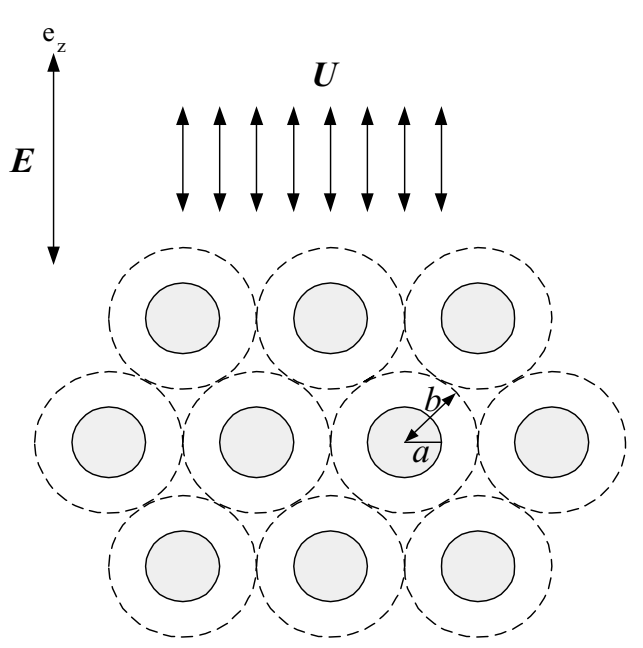

FIG. 1. Schematic representation of the system considered, which is represented by a representative particle of radius $a$ and a concentric liquid shell of radius $b . \mathbf{E}$ is the average dynamic electric field and $\mathbf{U}$ is the electrophoretic velocity.

tric field and time. Let $\mathbf{U}$ be the velocity of the representative particle $\mathbf{U}=U E_{Z} e^{-i \omega t} \mathbf{e}_{Z}$, where $U$ is the magnitude of $\mathbf{U}$, $U=U_{R}+i U_{I}, U_{R}$ and $U_{I}$ being respectively the real and the imaginary parts of $U$.

\subsection{Flow Field}

We assume that the liquid phase contains incompressible Newtonian fluid with constant physical properties and the flow field can be described by the Navier-Stokes equation in the creeping flow regime

$$
\begin{aligned}
\nabla \cdot \mathbf{u} & =0 \\
\rho_{f} \frac{\partial \mathbf{u}}{\partial t} & =-\nabla p+\eta \nabla^{2} \mathbf{u}-\rho_{c} \nabla \phi .
\end{aligned}
$$

In these expressions $\nabla$ is the gradient operator, $\mathbf{u}$ is fluid velocity, $\rho_{f}$ and $\eta$ are respectively the density and the viscosity of the fluid, $p$ is the pressure, $\rho_{c}$ is the space charge density, $\nabla^{2}$ is the Laplace operator, and $\phi$ is the electrical potential.

\subsection{Concentration Field}

The variation of the concentration of ionic species $j$ can be described by

$$
\begin{aligned}
\frac{\partial n_{j}}{\partial t} & =-\nabla \cdot \mathbf{f}_{j} \\
\mathbf{f}_{j} & =-D_{j}\left(\nabla n_{j}+\frac{n_{j} \hat{e} z_{j}}{k_{B} T} \nabla \phi\right)+n_{j} \mathbf{u}
\end{aligned}
$$

where $n_{j}, f_{j}$, and $D_{j}$ are respectively the concentration, the flux, and the diffusivity of ionic species $j, k_{B}$ is the Boltzmann constant, $\hat{e}$ is the elementary charge, and $T$ is the absolute temperature. Equation [3] represents the conservation of ions, 
and Eq. [4] states that the driving force for $\mathbf{f}_{j}$ comprises that contributed by the concentration gradient, the electrical potential gradient, and the flow of the liquid phase.

\subsection{Electrical Field}

Suppose that the electrical field can be described by the Poisson equation

$$
\nabla^{2} \phi=-\frac{\rho_{c}}{\varepsilon}=-\sum_{j=1}^{2} \frac{z_{j} \hat{e} n_{j}}{\varepsilon},
$$

where $\varepsilon$ is the permittivity of the liquid phase.

We assume that the applied electric field is weak, and each dependent variable can be expressed as the sum of its value in the absence of the applied electric field, i.e., the equilibrium value, and a perturbed term. Also, since the problem under consideration is $\varphi$-symmetric, we have

$$
\begin{aligned}
\phi(r, \theta, t) & =\phi_{e}(r)+\delta \phi(r, \theta) E_{Z} e^{-i \omega t} \\
n_{j}(r, \theta, t) & =n_{j}^{e}(r, \theta)+\delta n_{j}(r, \theta) E_{Z} e^{-i \omega t} \\
u(r, \theta, t) & =\mathbf{0}+\delta u(r, \theta) E_{Z} e^{-i \omega t} \\
p(r, \theta, t) & =p_{e}(r, \theta)+\delta p(r, \theta) E_{Z} e^{-i \omega t} .
\end{aligned}
$$

These expressions imply that the governing equation for a dependent variable can be decomposed into an equation describing its equilibrium state (i.e., state in the absence of the applied electric field) and that describing its perturbed state.

\subsection{Equilibrium State}

In the absence of the applied electric field $\mathbf{u}$ vanishes, and Eqs. [1] and [2] yield

$$
-D_{j}\left(\nabla n_{j}^{e}+\frac{n_{j}^{e} \hat{e} z_{j}}{k_{B} T} \nabla \phi_{e}\right)=0 .
$$

Integrating this expression, we obtain

$$
n_{j}^{e}=n_{j 0}^{e} \exp \left(-\frac{z_{j} \hat{e} \phi_{e}}{k_{B} T}\right)
$$

where $n_{j 0}^{e}$ is the bulk concentration of ionic species $j$. Substituting Eq. [5] into Eq. [11] gives

$$
\nabla^{* 2} \phi_{e}^{*}\left(r^{*}\right)=-\sum_{j=1}^{2} \frac{z_{j} \hat{e}^{2} n_{j 0}^{e} a^{2}}{\varepsilon k_{B} T} \exp \left(-z_{j} \phi_{e}^{*}\right),
$$

where $r^{*}=r / a$ and $\phi_{e}^{*}=\phi_{e} /\left(k_{B} T / \hat{e}\right)$.

The present cell model requires that a cell as a whole is electrically neutral, which implies that there is no net current across the virtual surface, $r=b$. Therefore,

$$
\frac{d \phi_{e}^{*}}{d r^{*}}=0, \quad r^{*}=b / a .
$$

We consider the following dissociation reaction on a particle surface:

$$
A \mathrm{H} \Leftrightarrow A^{-}+\mathrm{H}^{+} .
$$

The equilibrium constant $K_{a}$ can be expressed as

$$
K_{a}=\frac{\left[A^{-}\right]\left[\mathrm{H}^{+}\right]_{s}}{[A \mathrm{H}]},
$$

where a symbol with a square bracket represents concentration and $\left[\mathrm{H}^{+}\right]_{s}$ is the concentration of $\mathrm{H}^{+}$on a particle surface. We assume that the spatial variation of $\left[\mathrm{H}^{+}\right]$follows the Boltzmann distribution, that is,

$$
\left[\mathrm{H}^{+}\right]_{s}=\left[\mathrm{H}^{+}\right]_{0} \exp \left(\frac{-e \phi_{e}}{k_{B} T}\right)
$$

where $\left[\mathrm{H}^{+}\right]_{0}$ is the bulk concentration of $\mathrm{H}^{+}$. If we let $N_{s}$ be the concentration of the dissociable functional groups on a particle surface, then

$$
N_{s}=\left[A^{-}\right]+[A \mathrm{H}] .
$$

Combining Eqs. [15]-[17] gives

$$
\left[A^{-}\right]=\frac{N_{s}}{1+\left(\left[\mathrm{H}^{+}\right]_{0} / K_{a}\right) \exp \left(-e \phi_{e} / k_{B} T\right)} .
$$

If we let $\sigma$ be the charge density on a particle surface, then

$$
\sigma=-e\left[A^{-}\right] .
$$

Substituting Eq. [18] into Eq. [19] yields

$$
\sigma=-\frac{e N_{s}}{1+\left(\left[\mathrm{H}^{+}\right]_{0} / K_{a}\right) \exp \left(-e \phi_{e} / k_{B} T\right)} .
$$

Suppose that the permittivity of a liquid is much larger than that of a particle. Then, applying Gauss law yields

$$
\sigma=-\varepsilon\left(\frac{d \phi_{e}}{d r}\right)_{r=a}
$$

Substituting Eq. [20] into Eq. [21] gives

$$
\begin{aligned}
\frac{d \phi_{e}^{*}}{d r^{*}} & =\frac{e^{2} N_{s} a / \varepsilon k_{B} T}{1+\left(\left[\mathrm{H}^{+}\right]_{0} / K_{a}\right) \exp \left(-\phi_{e}^{*}\right)} \\
& =\frac{A}{1+B \exp \left(-\phi_{e}^{*}\right)}, \quad r^{*}=1,
\end{aligned}
$$

where $A=\hat{e}^{2} N_{s} a / \varepsilon k_{B} T$ and $B=\left[\mathrm{H}^{+}\right]_{0} / K_{a}$. 


\subsection{Perturbed State}

Since $\mathbf{u}$ vanishes at equilibrium state, Eqs. [1], [2], [8], and [9] lead to

$$
\begin{aligned}
\nabla \cdot\left(\delta \mathbf{u} E_{Z} e^{-i \omega t}\right) & =\mathbf{0} \\
-i \omega \rho_{f} \delta \mathbf{u} E_{Z} e^{-i \omega t} & =-\nabla \delta p E_{Z} e^{-i \omega t}+\eta \nabla^{2} \delta \mathbf{u} E_{Z} e^{-i \omega t}-\rho_{c} \nabla \phi .
\end{aligned}
$$

The pressure term in the last expression can be eliminated by introducing a stream function, $\psi$, representation. In terms of $\psi$ the magnitude of the perturbed velocity in the $r$-direction and that in $\theta$-direction, $\delta u_{r}$ and $\delta u_{\theta}$, can be represented respectively by

$$
\delta u_{r}=-\frac{1}{r^{2} \sin \theta} \frac{\partial \psi}{\partial \theta}
$$

and

$$
\delta u_{\theta}=\frac{1}{r \sin \theta} \frac{\partial \psi}{\partial r} .
$$

We have

$$
\delta \mathbf{u} E_{Z} e^{-i \omega t}=\left(\delta u_{r} \hat{\mathrm{r}}+\delta u_{\theta} \hat{\theta}\right) E_{Z} e^{-i \omega t} .
$$

Taking curl on both sides of Eq. [24] gives

$$
E^{4} \psi+i \omega \rho_{f} E^{2} \psi=\frac{1}{\eta}\left(\frac{\partial \rho_{c}}{\partial r} \frac{\partial \phi}{\partial \theta}-\frac{\partial \rho_{c}}{\partial \theta} \frac{\partial \phi}{\partial r}\right) \sin \theta,
$$

where

$$
\begin{aligned}
& E^{2}=\left(\frac{\partial^{2}}{\partial r^{2}}\right)+\frac{\sin \theta}{r^{2}} \frac{\partial}{\partial \theta}\left(\frac{1}{\sin \theta} \frac{\partial}{\partial \theta}\right) \\
& E^{4}=E^{2} E^{2}=\left(\left(\frac{\partial^{2}}{\partial r^{2}}\right)+\frac{\sin \theta}{r^{2}} \frac{\partial}{\partial \theta}\left(\frac{1}{\sin \theta} \frac{\partial}{\partial \theta}\right)\right)^{2} .
\end{aligned}
$$

Note that the concentration field is influenced by the perturbed flow field, which arises from the applied electric field, and therefore, the first two fields need to be considered simultaneously.

Taking $\nabla^{2}$ on both sides of Eq. [6] yields

$$
\nabla^{2}\left(\delta \phi(r, \theta) E_{Z} e^{-i \omega t}\right)=\nabla^{2} \phi(r, \theta, t)-\nabla^{2} \phi_{e}(r) .
$$

Similarly, Eq. [12] leads to

$$
\nabla^{2} \phi=-\sum_{j=1}^{2} \frac{z_{j} \hat{e} n_{j \mathbf{0}}^{e}}{\varepsilon} \exp \left(-\frac{z_{j} \hat{e}\left(\phi+g_{j} E_{Z} e^{-i \omega t}\right)}{k_{B} T}\right),
$$

where

$$
n_{j}=n_{j 0}^{e} \exp \left(-\frac{z_{j} \hat{e}\left(\phi+g_{j} E_{Z} e^{-i \omega t}\right)}{k_{B} T}\right) .
$$

This expression suggests that the electrical potential is related to the equilibrium potential, perturbed potential, and the induced potential, which arises from the concentration field. For con- venience, the concentration field is represented by $g_{j}$ through combining Eqs. [7], [11], and [33]. We have

$$
\begin{aligned}
\delta n_{j} E_{Z} e^{-i \omega t}= & n_{j 0}^{e}\left(\exp \left(-\frac{z_{j} \hat{e}\left(\phi+g_{j} E_{Z} e^{-i \omega t}\right)}{k_{B} T}\right)\right. \\
& \left.-\exp \left(-\frac{z_{j} \hat{e} \phi_{e}}{k_{B} T}\right)\right) .
\end{aligned}
$$

Combining Eqs. [3] and [4] gives

$\frac{\partial n_{j}}{\partial t}=-D_{j}\left(\nabla^{2} n_{j}+\frac{z_{j} \hat{e}}{k_{B} T}\left(\nabla n_{j} \cdot \nabla \phi+n_{j} \nabla^{2} \phi\right)\right)+\mathbf{u} \cdot \nabla n_{j}$.

Substituting Eqs. [6]-[8] into Eq. [35] yields

$$
\begin{aligned}
-i \omega \delta n_{j}= & -D_{j}\left(\nabla^{2}\left(\delta n_{j}\right)+\frac{z_{j} \hat{e}}{k T}\left(\nabla n_{j}^{e} \cdot \nabla\left(\delta n_{j}\right)+\nabla\left(\delta n_{j}\right) \cdot \nabla \phi_{e}\right.\right. \\
& +\nabla\left(\delta n_{j}\right) \cdot \nabla\left(\delta \phi E_{Z} e^{-i \omega t}\right)+n_{j}^{e} \nabla^{2}(\delta \phi) \\
& \left.\left.+\left(\delta n_{j}\right) \nabla^{2} \phi_{e}+\left(\delta n_{j}\right) \nabla^{2}\left(\delta \phi E_{Z} e^{-i \omega t}\right)\right)\right) \\
& +(\delta u) \cdot \nabla\left(n_{j}^{e}+\delta n_{j} E_{Z} e^{-i \omega t}\right) .
\end{aligned}
$$

For a simpler mathematical treatment, Eqs. [28] and [32]-[36] are linearized to yield the following governing equations for the perturbed variables:

$$
\begin{aligned}
& \nabla^{* 2} \delta \phi^{*}-\frac{(\kappa a)^{2}}{1+\alpha}\left[\exp \left(-\phi_{r} \phi_{e}^{*}\right)+\alpha \exp \left(\alpha \phi_{r} \phi_{e}^{*}\right)\right] \delta \phi^{*} \\
& =\frac{(\kappa a)^{2}}{1+\alpha}\left[\exp \left(-\phi_{r} \phi_{e}^{*}\right) g_{1}^{*}+\alpha \exp \left(\alpha \phi_{r} \phi_{e}^{*}\right) g_{2}^{*}\right] \\
& \nabla^{* 2} g_{1}^{*}-\phi_{r} \nabla^{*} \phi_{e}^{*} \cdot \nabla^{*} g_{1}^{*}+P e_{1} \delta \mathbf{u}^{*} \cdot \nabla^{*} \phi_{e}^{*} \\
& +i \frac{\omega a^{2}}{D_{1}}\left(\delta \phi^{*}+g_{1}^{*}\right)=0 \\
& \nabla^{* 2} g_{2}^{*}+\alpha \phi_{r} \nabla^{*} \phi_{e}^{*} \cdot \nabla^{*} g_{2}^{*}+P e_{2} \delta \mathbf{u}^{*} \cdot \nabla^{*} \phi_{e}^{*} \\
& \quad+i \frac{\omega a^{2}}{D_{2}}\left(\delta \phi^{*}+g_{2}^{*}\right)=0
\end{aligned}
$$

$E^{* 4} \psi^{*}+i \frac{\rho_{f} \omega a^{2}}{\eta} E^{* 2} \psi^{*}$

$$
=\frac{(\kappa a)^{2}}{1+\alpha}\left[\frac{\partial g_{1}^{*}}{\partial \theta} \exp \left(-\phi_{r} \phi_{e}^{*}\right)+\alpha \frac{\partial g_{2}^{*}}{\partial \theta} \exp \left(\alpha \phi_{r} \phi_{e}^{*}\right)\right] \frac{\partial \phi_{e}^{*}}{\partial r^{*}} \sin \theta \text {. }
$$

The scaled quantities are $E_{Z}^{*}=E_{Z} /(\zeta / a), U^{*} E_{Z}^{*} e^{-i \omega t}=$ $U E_{Z} e^{-i \omega t} / U_{E}, \quad \phi_{e}^{*}=\phi_{e} / \zeta, \quad \delta \phi^{*} E_{Z}^{*} e^{-i \omega t}=\delta \phi E_{Z} e^{-i \omega t} / \zeta$, $g_{j}^{*} E_{Z}^{*} e^{-i \omega t}=g_{j} E_{Z} e^{-i \omega t} / \zeta, \quad \psi^{*} E_{Z}^{*} e^{-i \omega t}=\psi E_{Z} e^{-i \omega t} / U_{E} a$, $n_{j}^{*}=n_{j} / n_{10}^{e}$, and $r^{*}=r / a$. In these expressions, $\kappa=\left(\varepsilon k_{B} T /\right.$ $\left.\sum_{j=1}^{2} n_{j 0}^{e}\left(\widehat{e} z_{j}\right)^{2}\right)^{-1 / 2}$ is the reciprocal Debye length, $P e_{j}=$ $U_{E} a^{2} / D_{j}, j=1,2$, is the electric Peclet number of ionic 
species $j$, and $\left(\rho_{f} \omega a^{2} / \eta\right)$ and $\left(\omega a^{2} / D_{j}\right), j=1,2$, are scaled frequencies.

A complex number is introduced to facilitate the analysis of the oscillatory phenomena involved in the electroacoustics. Both the magnitude of the dynamic electrophoretic velocity and the phase angle can be easily represented based on the complex notation (22). Moreover, the geometry of the system under consideration justifies the application of the separation of variables technique. As a result, Eqs. [37]-[40] can be rewritten in complex form as

$$
\begin{aligned}
\delta \phi^{*} & =\left(\Phi_{R}+i \Phi_{I}\right) \cos \theta \\
g_{1}^{*} & =\left(G_{1 R}+i G_{1 I}\right) \cos \theta \\
g_{2}^{*} & =\left(G_{2 R}+i G_{2 I}\right) \cos \theta \\
\psi^{*} & =\left(\Psi_{R}+i \Psi_{I}\right) \sin ^{2} \theta,
\end{aligned}
$$

where the complex variables in the parentheses are functions of $r$ only. Substituting Eqs. [41]-[44] into Eqs. [37]-[40], we obtain

$$
\begin{aligned}
& L_{1}^{2} \Phi_{R}-\frac{(\kappa a)^{2}}{1+\alpha}\left[\exp \left(-\phi_{r} \phi_{e}^{*}\right)+\alpha \exp \left(\alpha \phi_{r} \phi_{e}^{*}\right)\right] \Phi_{R} \\
& =\frac{(\kappa a)^{2}}{1+\alpha}\left[\exp \left(-\phi_{r} \phi_{e}^{*}\right) G_{1 R}+\alpha \exp \left(\alpha \phi_{r} \phi_{e}^{*}\right) G_{2 R}\right] \\
& L_{1}^{2} \Phi_{I}-\frac{(\kappa a)^{2}}{1+\alpha}\left[\exp \left(-\phi_{r} \phi_{e}^{*}\right)+\alpha \exp \left(\alpha \phi_{r} \phi_{e}^{*}\right)\right] \Phi_{I} \\
& =\frac{(\kappa a)^{2}}{1+\alpha}\left[\exp \left(-\phi_{r} \phi_{e}^{*}\right) G_{1 I}+\alpha \exp \left(\alpha \phi_{r} \phi_{e}^{*}\right) G_{2 I}\right] \\
& L_{1}^{2} G_{1 R}-\phi_{r} \frac{d \phi_{e}^{*}}{d r^{*}} \frac{d G_{1 R}}{d r^{*}}+P e_{1} \frac{1}{r^{* 2}} \Psi_{R} \frac{d \phi_{e}^{*}}{d r^{*}} \\
& -\frac{\omega a^{2}}{D_{1}}\left(\Phi_{I}+G_{1 I}\right)=0
\end{aligned}
$$

$$
\begin{gathered}
L_{1}^{2} G_{1 I}-\phi_{r} \frac{d \phi_{e}^{*}}{d r^{*}} \frac{d G_{1 I}}{d r^{*}}+P e_{1} \frac{1}{r^{* 2}} \Psi_{I} \frac{d \phi_{e}^{*}}{d r^{*}} \\
+\frac{\omega a^{2}}{D_{1}}\left(\Phi_{R}+G_{1 R}\right)=0
\end{gathered}
$$

$$
\begin{aligned}
& L_{1}^{2} G_{2 R}+\alpha \phi_{r} \frac{d \phi_{e}^{*}}{d r^{*}} \frac{d G_{2 R}}{d r^{*}}+P e_{2} \frac{1}{r^{* 2}} \Psi_{R} \frac{d \phi_{e}^{*}}{d r^{*}} \\
& -\frac{\omega a^{2}}{D_{2}}\left(\Phi_{I}+G_{2 I}\right)=0
\end{aligned}
$$

$$
\begin{aligned}
& L_{1}^{2} G_{2 I}+\alpha \phi_{r} \frac{d \phi_{e}^{*}}{d r^{*}} \frac{d G_{2 I}}{d r^{*}}+P e_{2} \frac{1}{r^{* 2}} \Psi_{I} \frac{d \phi_{e}^{*}}{d r^{*}} \\
& +\frac{\omega a^{2}}{D_{2}}\left(\Phi_{R}+G_{2 R}\right)=0
\end{aligned}
$$

$$
\begin{aligned}
L_{2}^{4} \Psi_{R}-\frac{\rho_{f} \omega a^{2}}{\eta} L_{2}^{2} \Psi_{I}= & -\frac{(\kappa a)^{2}}{(1+\alpha)}\left(G_{1 R} \exp \left(-\phi_{r} \phi_{e}^{*}\right)\right. \\
& \left.+\alpha G_{2 R} \exp \left(\alpha \phi_{r} \phi_{e}^{*}\right)\right) \frac{d \phi_{e}^{*}}{d r^{*}}
\end{aligned}
$$

$$
\begin{aligned}
L_{2}^{4} \Psi_{I}+\frac{\rho_{f} \omega a^{2}}{\eta} L_{2}^{2} \Psi_{R}= & -\frac{(\kappa a)^{2}}{(1+\alpha)}\left(G_{1 I} \exp \left(-\phi_{r} \phi_{e}^{*}\right)\right. \\
& \left.+\alpha G_{2 I} \exp \left(\alpha \phi_{r} \phi_{e}^{*}\right)\right) \frac{d \phi_{e}^{*}}{d r^{*}}
\end{aligned}
$$

where

$$
L_{2}^{4}=L_{2}^{2} L_{2}^{2}
$$

and

$$
\begin{aligned}
L_{1}^{2} & \equiv \frac{d^{2}}{d r^{* 2}}+\frac{2}{r^{* 2}} \frac{d}{d r^{*}}-\frac{2}{r^{* 2}} \\
L_{2}^{2} & \equiv \frac{d^{2}}{d r^{* 2}}-\frac{2}{r^{* 2}} .
\end{aligned}
$$

Note that due to the introduction of the complex notation, the number of equations is doubled.

We assume the following: (a) The surface of a particle is impenetrable to ions. (b) The potential on a cell surface arises from the applied electric field (22). (c) The perturbed concentration vanishes on a cell surface. (d) No-slip conditions exist on a particle surface. (e) The vorticity vanishes on a cell surface. (f) There is no net flow in the radial direction across a cell surface. Therefore, the boundary conditions associated with Eqs. [45]-[52] are

$$
\begin{aligned}
& \frac{d \Phi_{R}}{d r^{*}}=0, \quad r^{*}=1 \\
& \Phi_{R}=-E_{Z}^{*} \frac{b}{a}, \quad r^{*}=b / a \\
& \frac{d \Phi_{I}}{d r^{*}}=0, \quad r^{*}=1 \\
& \Phi_{I}=-E_{Z}^{*} \frac{b}{a}, \quad r^{*}=b / a \\
& \frac{d G_{1 R}}{d r^{*}}=0, \quad r^{*}=1 \\
& G_{1 R}=-\Phi_{R}, \quad r^{*}=b / a \\
& \frac{d G_{1 I}}{d r^{*}}=0, \quad r^{*}=1 \\
& G_{1 I}=-\Phi_{I}, \quad r^{*}=b / a \\
& \frac{d G_{2 R}}{d r^{*}}=0, \quad r^{*}=1 \\
& G_{2 R}=-\Phi_{R}, \quad r^{*}=b / a \\
& \frac{d G_{2 I}}{d r^{*}}=0, \quad r^{*}=1 \\
& G_{2 I}=-\Phi_{I}, \quad r^{*}=b / a \\
& \frac{\Psi_{R}}{d \Psi_{R}}=\frac{1}{2} U_{R}^{*} r^{* 2}, \quad r^{*}=1 \\
& r_{R}^{*} r^{*}, \quad r^{*}=1
\end{aligned}
$$




$$
\begin{aligned}
L_{2}^{2} \Psi_{R} & =0, \quad r^{*}=b / a \\
\Psi_{R} & =0, \quad r^{*}=b / a \\
\Psi_{I} & =\frac{1}{2} U_{I}^{*} r^{* 2}, \quad r^{*}=1 \\
\frac{d \Psi_{I}}{d r^{*}} & =U_{I}^{*} r^{*}, \quad r^{*}=1 \\
L_{2}^{2} \Psi_{I} & =0, \quad r^{*}=b / a \\
\Psi_{I} & =0, \quad r^{*}=b / a .
\end{aligned}
$$

Note again that the number of boundary conditions is doubled due to the introduction of the complex notation. The details of these derivations can be found elsewhere (22).

\subsection{Electrophoretic Mobility}

For convenience, the problem under consideration is decomposed into two subproblems: a particle moves with velocity $\left(U_{R}^{*}+i U_{I}^{*}\right) E_{Z}^{*} e^{-i \omega t}$ in the absence of the applied electric field and the particle remains fixed when an electric field $E_{Z}^{*} e^{-i \omega t}$ is applied. The force experienced by the particle is the sum of the hydrodynamic force, $\mathbf{F}_{h}$, and the electric force $\mathbf{F}_{e}$. In scaled
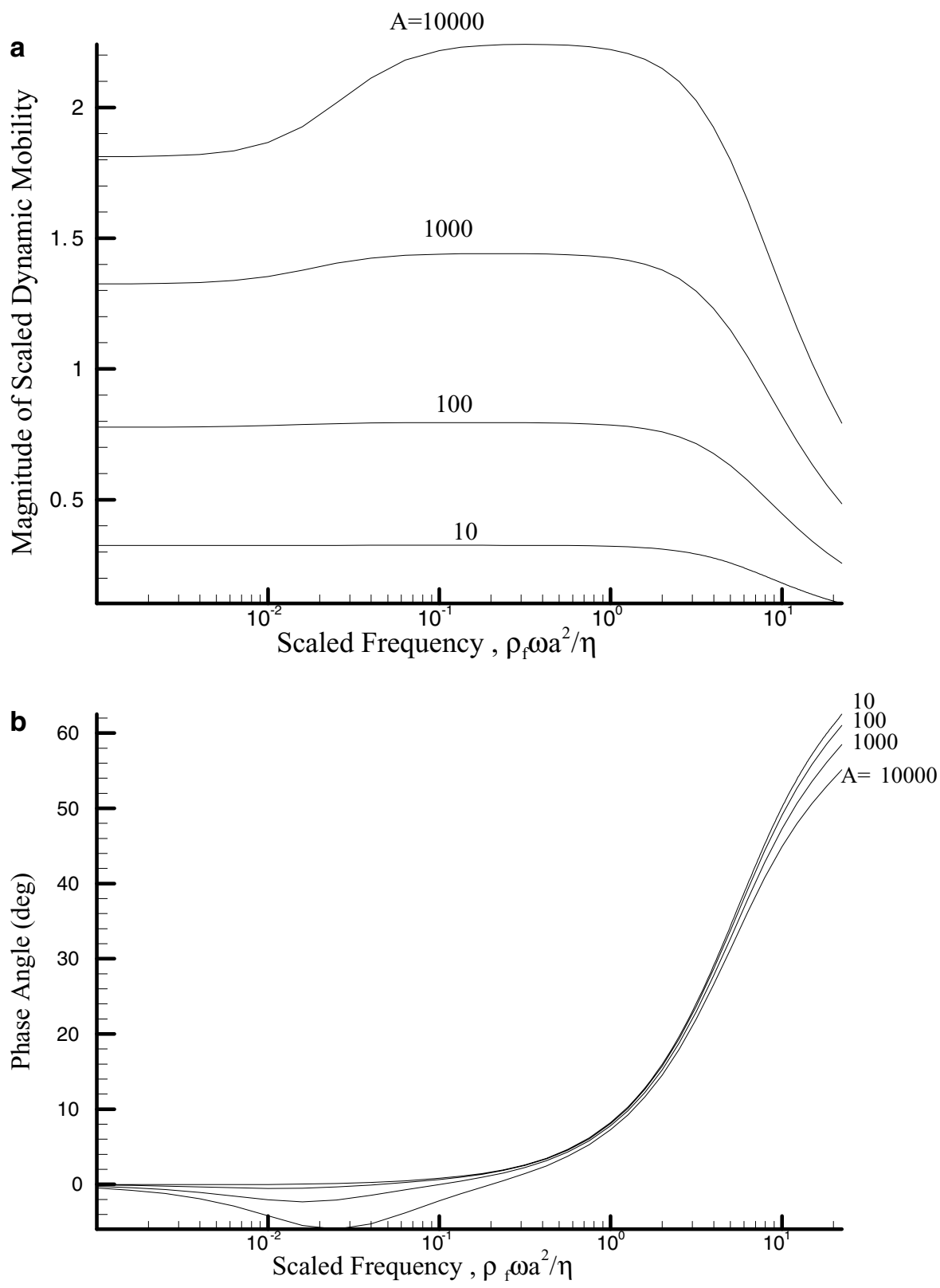

FIG. 2. Variation of magnitude of scaled dynamic mobility, (a), and phase angle, (b), as a function of scaled frequency $\left(\rho_{f} \omega a^{2} / \eta\right)$ at various $A$ for the case $B=1.0, \kappa a=1.0$, and $\lambda=a / b=0.5$. 
form, we have

$$
\begin{aligned}
F_{h}^{*}+F_{e}^{*}= & -i\left(\frac{4}{3} \pi \varepsilon \zeta^{2}\right)\left(\frac{\rho_{f} \omega a^{2}}{\eta}\right)\left(\frac{\rho_{p}-\rho_{f}}{\rho_{f}}\right) \\
& \times\left(U_{R}^{*}+i U_{I}^{*}\right) E_{Z}^{*} e^{-i \omega t},
\end{aligned}
$$

where $F_{h}^{*}$ and $F_{e}^{*}$ are respectively the magnitudes of the scaled hydrodynamic force, $F_{h}^{*}$, and the electrical force, $F_{e}^{*}$, exerted on a particle. $F_{h}^{*}$ and $F_{e}^{*}$ can be evaluated respectively by

$$
F_{e}^{*}=\frac{8}{3} \pi \varepsilon \zeta^{2}\left[r^{*} \frac{d \phi_{e}^{*}}{d r^{*}}\left(\Phi_{R}+i \Phi_{I}\right) E_{Z}^{*} e^{-i \omega t}\right]_{r^{*}=1}
$$

and

$$
\begin{aligned}
F_{h}^{*}= & \frac{4}{3} \pi \varepsilon \zeta^{2}\left[r^{* 4} \frac{\partial}{\partial r^{*}}\left(\frac{L_{2}^{2}\left(\Psi_{R}+i \Psi_{I}\right)}{r^{* 2}}\right) E_{z}^{*} e^{-i \omega t}\right]_{r^{*}=1} \\
& +\frac{4}{3} \pi \varepsilon \zeta^{2}\left[r^{* 2}\left(\exp \left(-\phi_{r} \phi_{e}^{*}\right)-\exp \left(\alpha \phi_{r} \phi_{e}^{*}\right)\right)\right. \\
& \left.\times\left(\Phi_{R}+i \Phi_{I}\right) E_{z}^{*} e^{-i \omega t}\right]_{r^{*}=1} .
\end{aligned}
$$

The electrophoretic velocity of a particle can be calculated based on Eqs. [76]-[78].

\section{RESULTS AND DISCUSSION}

Figure 2 shows both the variations of the magnitude and the phase angle of the scaled dynamic mobility as a function of
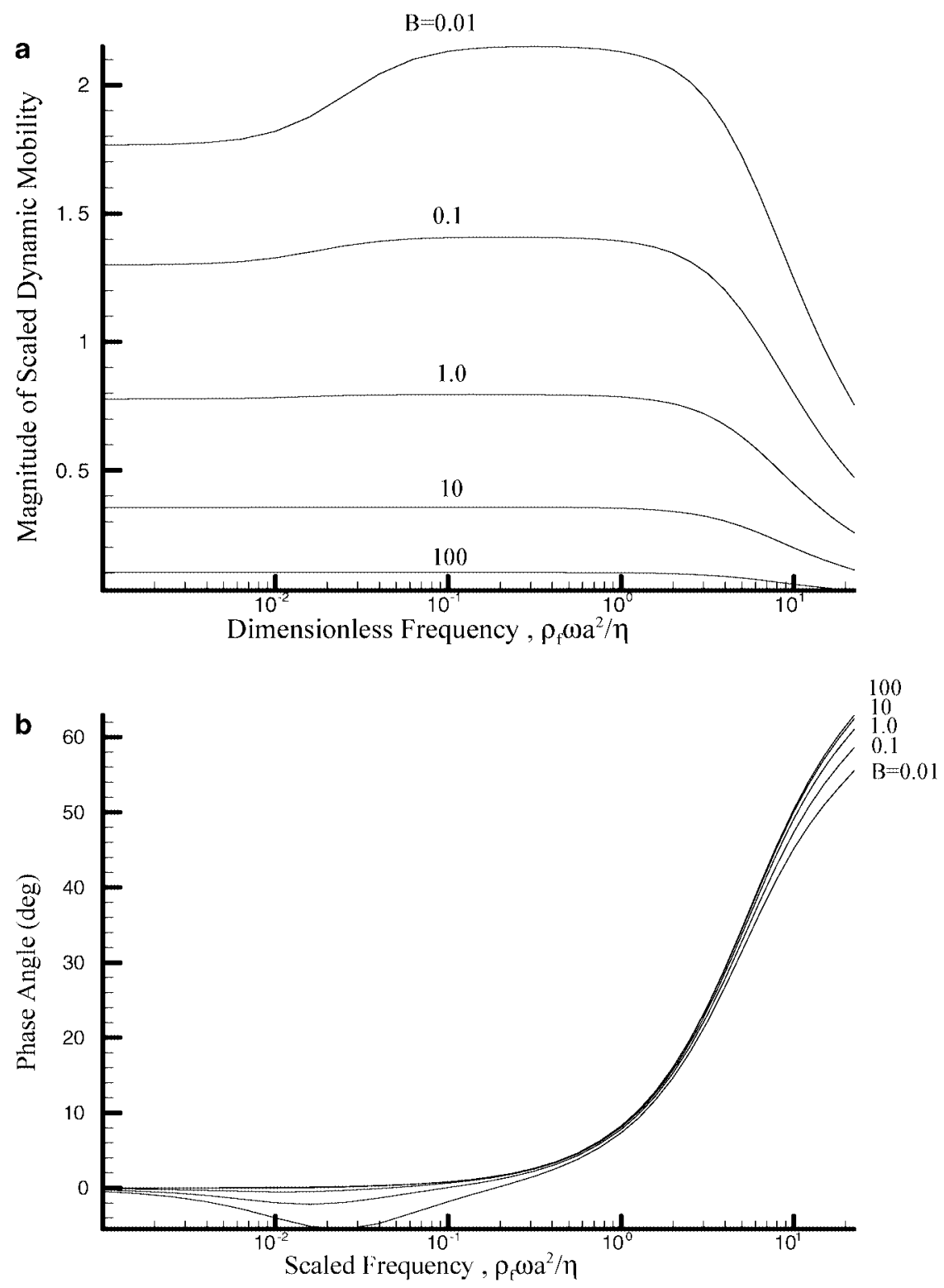

FIG. 3. Variation of magnitude of scaled dynamic mobility, (a), and phase angle, (b), as a function of scaled frequency $\left(\rho_{f} \omega a^{2} / \eta\right)$ at various $B$ for the case $A=100, \kappa a=1.0$, and $\lambda=a / b=0.5$. 
the scaled frequency of the average electric field $\left(\rho_{f} \omega a^{2} / \eta\right)$ at various $A\left(=\hat{e}^{2} N_{s} a / \varepsilon k_{B} T\right)$. Figure $2 \mathrm{a}$ reveals that for a fixed $\left(\rho_{f} \omega a^{2} / \eta\right)$ the larger the $A$, the greater the magnitude of the scaled dynamic mobility. This is because a large $A$ implies a high concentration of the dissociable functional groups on a particle surface, which leads to a high surface potential. Figure 2a also indicates that if $A$ is small, the magnitude of the scaled dynamic mobility decreases with the increase in $\left(\rho_{f} \omega a^{2} / \eta\right)$. However, if $A$ is sufficiently large, the magnitude of the scaled dynamic mobility exhibits a local maximum at a medium value of $\left(\rho_{f} \omega a^{2} / \eta\right)$. This is mainly due to the phenomenon of double-layer polariza- tion, which is significant if the surface potential is sufficiently high. Double-layer polarization has the effect of inducing an internal electric field, which is in the inverse direction as that of the applied electric field. In this case, as the direction of the average electric field varies, since the induced electric field cannot respond instantaneously, it has the effect of accelerating the movement of a particle. However, if the frequency of the average electric field is high, the rate of change in the direction of the movement of the particle is fast. In this case it moves back and forth too fast, and increasing $\left(\rho_{f} \omega a^{2} / \eta\right)$ has a negative effect on the dynamic mobility. Note that $\left(\rho_{f} \omega a^{2} / \eta\right)$ can be interpreted
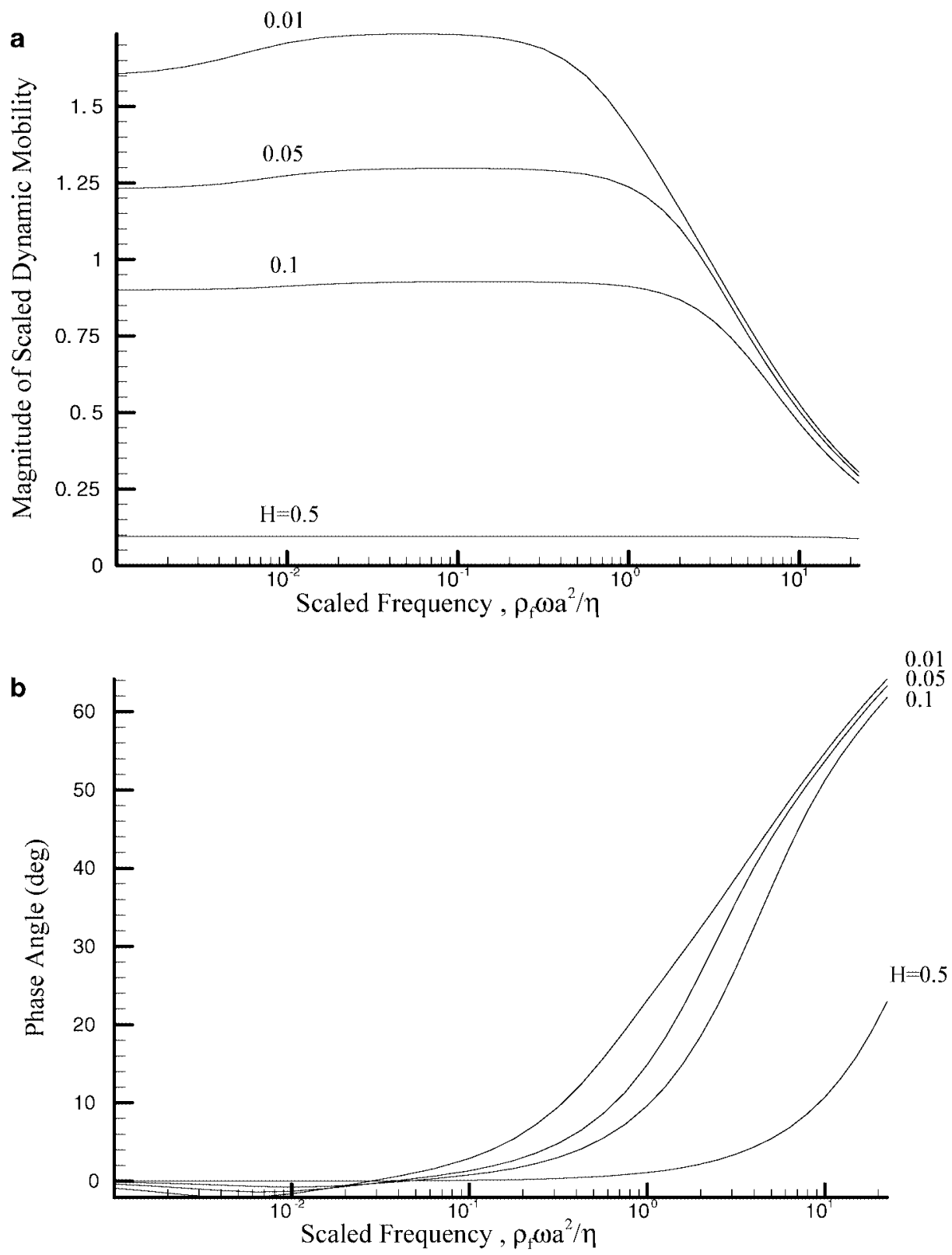

FIG. 4. Variation of magnitude of scaled dynamic mobility, (a), and phase angle, (b), as a function of scaled frequency $\left(\rho_{f} \omega a^{2} / \eta\right)$ at various $H$ for the case $A=100, B=1.0$, and $\kappa a=1.0$. 
as the ratio (dynamic inertia effect/viscous effect), which is a measure for the rate of response of the induced electric field to that of the average electric field. Figure $2 b$ reveals that if $A$ is small, the phase angle of the dynamic mobility is positive; that is, the average electric field leads the dynamic mobility, and the greater the $\left(\rho_{f} \omega a^{2} / \eta\right)$, the larger the phase angle. However, if $A$ is sufficiently large, the phase angle is negative (i.e., the dynamic mobility leads the average electric field) and has a negative local minimum as $\left(\rho_{f} \omega a^{2} / \eta\right)$ varies. Again, this is due to the effect of double-layer polarization.

The variations of the magnitude and the phase angle of the scaled dynamic mobility and as a function of the scaled frequency of the average electric field $\left(\rho_{f} \omega a^{2} / \eta\right)$ at various $B\left(=\left[\mathrm{H}^{+}\right]_{0} / K_{a}\right)$ are presented in Fig. 3. Figure 3a suggests that for a fixed $\left(\rho_{f} \omega a^{2} / \eta\right)$ the magnitude of the scaled dynamic mobility increases with the decrease in $B$. This is because the smaller the $B$, the larger the equilibrium constant $K_{a}$, which implies a higher charge density on a particle surface. Figure $3 \mathrm{a}$ also reveals that if $B$ is large, the magnitude of the scaled dynamic mobility decreases with the increase in $\left(\rho_{f} \omega a^{2} / \eta\right)$. However, if it is small, the magnitude of the scaled dynamic mobility has a local maximum as $\left(\rho_{f} \omega a^{2} / \eta\right)$ varies. These can be explained by the same reasoning as that in the discussion of Fig. 2a. Similarly, the qualitative behaviors of the phase angle shown in Fig. $3 \mathrm{~b}$ can be explained by the same reasoning as that in the discussion of Fig. 2b.

Figure 4 illustrates both the variations of the magnitude and the phase angle of the scaled dynamic mobility as a function of the scaled frequency of the average electric field $\left(\rho_{f} \omega a^{2} / \eta\right)$ at various $H\left(=(a / b)^{3}\right)$. Figure 4 a shows that for a fixed $\left(\rho_{f} \omega a^{2} / \eta\right)$ the larger the $H$, the smaller the magnitude of the scaled dynamic mobility. This is because the larger the $H$, the higher the concentration of particle, and the more significant the hindrance of neighboring particles, which leads to a smaller dynamic mobility. Figure 4a indicates that if $H$ is small, the magnitude of the scaled dynamic mobility has a local maximum as $\left(\rho_{f} \omega a^{2} / \eta\right)$ varies; the phase angle has a negative (i.e., phase lead) local minimum, as shown in Fig. 4b. Again, these are due to the effect of double-layer polarization. As can be seen in Fig. 4 if $H$ is large, both the local maximum in the magnitude of the scaled mobility and the local minimum in the phase angle become inappreciable. This is because if $H$ is large, the polarization of the double layer surrounding a particle is confined by the neighboring particles, and therefore its effect becomes relatively insignificant. For the same reason, the lag in the phase angle for the case where $H$ is large is smaller than the lag for the case where $H$ is small, as can be seen in Fig. 4b, since it is relatively easy for the particle to respond to the variation in the applied electric field.

Figure 5 shows the variation of the magnitude of the scaled dynamic mobility as a function of double-layer thickness $\kappa a$ at various scaled frequency of the average electric field $\left(\rho_{f} \omega a^{2} / \eta\right)$. It was found that, for the case of a static electric field, the mobility has a local maximum for $\kappa a$ in the range $[1,10](26)$. This is because if the double layer is thick, the resistant force expe-

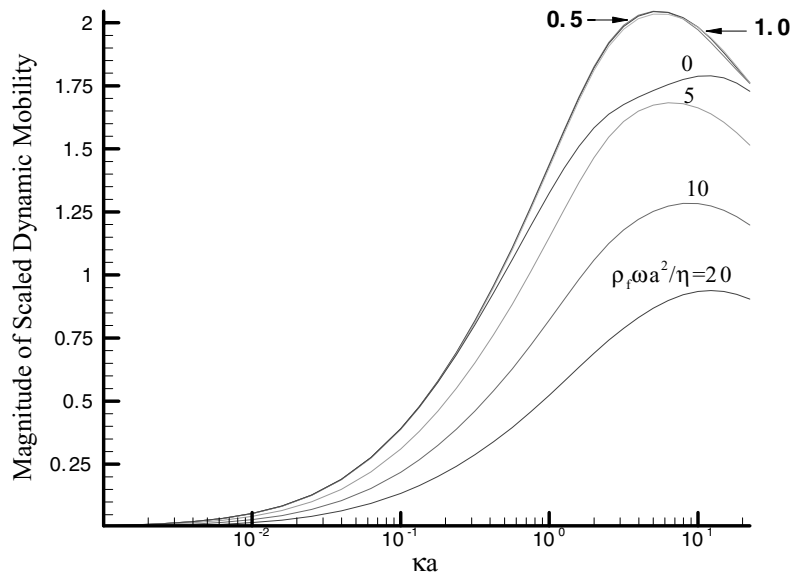

FIG. 5. Variation of magnitude of scaled dynamic mobility as a function of $\kappa a$ at various scaled frequency $\left(\rho_{f} \omega a^{2} / \eta\right)$ for the case $A=1000, B=1.0$, and $\lambda=a / b=0.5$.

rienced by a particle is large, which yields a small mobility. On the other hand, a thin double layer has the effect of confining the degree of dissociation of functional groups, which leads to a low surface charge density, and therefore, a small mobility. As can be seen in Fig. 5, a similar result to that observed in the case of a static electric field is obtained for the present case of a dynamic electric field. This figure also reveals that the local maximum of the scaled dynamic mobility increases first with the increase in $\left(\rho_{f} \omega a^{2} / \eta\right)$, reaches a maximum, and then decreases with a further increase in $\left(\rho_{f} \omega a^{2} / \eta\right)$. As mentioned previously, the effect of double-layer polarization can be advantageous to the movement of a particle if a dynamic electric field of appropriate frequency is applied. However, if $\left(\rho_{f} \omega a^{2} / \eta\right)$ is high, the polarization of a double layer is insignificant, and therefore, the acceleration on the movement of the particle which arises from the induced electric field becomes unimportant. The observation that the maximum in the maximal scaled dynamic mobility occurs at $\left(\rho_{f} \omega a^{2} / \eta\right) \cong 0.5$ is consistent with previous discussion.

\section{CONCLUSION}

The dynamic electrophoretic mobility of a concentrated dispersion of charge-regulated particles is investigated. The dynamic mobility of a particle is found to depend on two key parameters, $A$ and $B$, which characterize respectively the concentration and the degree of dissociation of the functional groups on a particle surface. If $A$ is large and/or $B$ is small, the absolute surface potential of the particle is high, and the effect of doublelayer polarization becomes significant. In this case the dynamic mobility may have a local maximum, and the phase angle may have a negative (phase lead) local minimum as the thickness of double layer varies. Due the hindrance of neighboring particles, the dynamic mobility decreases with the increase in the volume fraction of a particle. 


\section{ACKNOWLEDGMENT}

This work is supported by the National Science Council of the Republic of China.

\section{REFERENCES}

1. von Smoluchowski, M., Z. Phys. Chem., Stoechiom. Verwandtschaftsl. 92, 129 (1918).

2. Booth, F., Proc. R. Soc. London Ser. A 203, 514 (1950).

3. O'Brien, R. W., and White, L. R., J. Chem. Soc. Faraday Trans. 2 74, 1607 (1978).

4. Kozak, M. W., and Davis, E. J., J. Colloid Interface Sci. 127, 497 (1989).

5. Kozak, M. W., and Davis, E. J., J. Colloid Interface Sci. 129, 166 (1989).

6. O’Brien, R. W., J. Fluid Mech. 190, 71 (1988).

7. Show, D. J., in "Introduction to Colloid and Surface Chemistry," 4th ed., pp. 174-199. Butterworth Press, Oxford, 1992.

8. Babchin, A. J., Chow, R. S., and Sawatzky, R. P., Adv. Colloid Interface Sci. 30, 111 (1989).

9. Sawatzky, R. P., and Babchin, A. J., J. Fluid Mech. 246, 321 (1993).

10. Fixman, M., J. Chem. Phys. 78, 1483 (1983).
11. James, R. O., Texter, J., and Scales, P. J., Langmuir 7, 1993 (1991).

12. Mangelsdorf, C. S., and White, L. R., J. Chem. Soc. Faraday Trans. 88, 3567 (1992).

13. Mangelsdorf, C. S., and White, L. R., J. Colloid Interface Sci. 160, 275 (1993).

14. Ohshima, H., J. Colloid Interface Sci. 179, 431 (1996).

15. Ohshima, H., J. Colloid Interface Sci. 195, 137 (1997).

16. Ohshima, H., Colloids Surf. A 149, 5 (1999).

17. Ohshima, H., Colloids Surf. A 159, 293 (1999).

18. Kuwabara, S., J. Phys. Soc. Jpn. 14, 527 (1959).

19. Levine, S., and Neale, G. H., J. Colloid Interface Sci. 47, 520 (1974).

20. Dukhin, A. S., Shilov, V., and Borkovskaya, Y., Langmuir 15, 3452 (1999).

21. Shilov, V. N., Zharkikh, N. I., and Borkovskaya, Y. B., Colloid J. 43, 434 (1981).

22. Lee, E., Yen, F. Y., and Hsu, J. P., J. Phys. Chem. B 105, 7239 (2001).

23. Lee, E., Chu, J. W., and Hsu, J. P., J. Colloid Interface Sci. 205, 65 (1998).

24. Lee, E., Chu, J. W., and Hsu, J. P., J. Colloid Interface Sci. 209, 240 (1998).

25. Lee, E., Yen, F. Y., and Hsu, J. P., Electrophoresis 21, 475 (2000).

26. Hsu, J. P., Lee, E., and Yen, F. Y., J. Chem. Phys. 112, 6404 (2000).

27. Ninham, B. W., and Parsegian, V. A., J. Theor. Biol. 31, 405 (1971). 